Research Article

\title{
Evaluation of Antifingerprint Properties of Plastic Surfaces Used in Automotive Components
}

\author{
M. P. Luda $\left(\mathbb{D},{ }^{1}\right.$ N. Li Pira, ${ }^{2}$ D. Trevisan, ${ }^{1}$ and V. Pau ${ }^{1}$ \\ ${ }^{1}$ Dipartimento di Chimica, Università di Torino, Via P. Giuria 7 10125, Torino, Italy \\ ${ }^{2}$ GML, C.R.F. S.C.p.A, Corso Settembrini 40, 10135 Torino, Italy \\ Correspondence should be addressed to M. P. Luda; mariapaola.luda@unito.it
}

Received 17 May 2018; Revised 21 September 2018; Accepted 24 October 2018; Published 28 November 2018

Academic Editor: Stefano Turri

Copyright (C) 2018 M. P. Luda et al. This is an open access article distributed under the Creative Commons Attribution License, which permits unrestricted use, distribution, and reproduction in any medium, provided the original work is properly cited.

\begin{abstract}
The antifingerprint properties of a range of surfaces produced with different technologies (in-mould decoration, in-mould labeling, and painted) were objectively evaluated by depositing on them in standard conditions an artificial fingerprint for direct determination of its visibility. The artificial fingerprint behaves similarly to the real human fingerprints. A classification method is then proposed to classify surfaces on the base of antifingerprint properties by measuring the roughness profile (Ra) and calculating the \% variation of gloss (GU 20 and $60^{\circ}$ ), haze, luminance $(L)$, and diffuse reflectance $(R)$ values after fingerprint deposition. This approach provides an objective and quantitative test method to determine visual antifingerprint properties of coated surfaces, instead of the "easy-to-clean" properties commonly evaluated. The data acquired provides a design guideline for fabricating visually fingerprint-free surfaces by controlling roughness, texture, color, and transparency of surfaces, with the aim of optically masking fingerprints.
\end{abstract}

\section{Introduction}

Today, most research is focused on improving easy-to-clean properties and self-cleaning (antistaining, antisoiling) properties of the surfaces used in most industrial sectors [1]. In particular, these properties are very important on surfaces touched and manipulated by the user such as touch screen displays since fingerprints easily stick on them; as a consequence, their optical quality is impaired and information becomes quite difficult to read. For this reason, the fingerprint problem on surfaces is a pressing issue not only in the automotive sector. In most situations, antifingerprint properties are required on surfaces resulting from new technologies such as in-mould decoration (IMD) and in-mould labelling (IML), alternatives of the conventional injection molding process. In IMD, a molten polymer is introduced into the mould cavity where a film carrying the layers of dried paint, decorated with heat-resistant ink, is attached to one side of the mould walls with the paint facing the gate. The paint is transferred to the plastic, and the carrier film is removed when opening the mold. IMD has been employed for the production of various molded products such as automotive interior parts, mobile phone cases, and logoimprinted plastic products. IML utilizes plastic labels, made with various polymers; the labels become an integral part of the final product, which is delivered as a predecorated item. IML products include picnic ware, mouse mats, or internal automotive components. The development of new effective products with controlled characteristics depends strictly on the knowledge of what governs the antifingerprint properties of the surfaces.

Fingerprints possess amphiphobic properties [2-4], so an appropriate surface roughness and surface chemistry are both crucial factors in the fabrication of fingerprintfree surfaces. Main top-down methods for preparation of superoleophobic plastic surfaces are etching, lithography, and laser processing whilst main bottom-up methods are electrodeposition, hydrothermal method, electrospinning, and sol-gel process [5]. Other bottom-up methods for superoleophobic surfaces have also been reported, such as 
spray deposition, dip coating, self-assembly, physical vapor deposition (PVD), and the template method. Though all these methods can be applied to fabricate superoleophobic surfaces, few can be used in industrial production because of their high cost and limited production volumes and the inherent complexity of the manufacturing process. So far, many companies carry out applications of hydrophobic and oleophobic coatings to automotive display manufacturers both on plastic and on glass-based displays [6]. The amphiphobic property can also be achieved in a nanoscaled concave structure consisting of cavities, in which the capillary force produced at the liquid-air interface inside the cavity is able to repel the entering liquid (water or oil) [7].

However, imparting antifingerprint properties to surfaces is complex because of fingerprint composition, which depends on the time elapsed since deposition, on the gender, on the age, and on the diet of the person touching the surface $[3,4]$. Consequently, successful antifingerprint properties cannot be easily predicted from the resins used or material labels alone.

Currently, the evaluation of the visibility of fingerprints on surfaces is only visual (with the naked eye) so an objective test method is required. To our knowledge, the only method found in literature able to evaluate the behavior of surfaces against fingerprints is the one proposed by $\mathrm{Wu}$ et al. [8] who measured the contact angles on smooth and rough surfaces prepared on purpose by sol-gel technique using an artificial fingerprint liquid. This method suggests that a surface with a contact angle above $87^{\circ}$ is virtually fingerprint free.

Aside from the fact that the abovementioned method works or not, it is evident that it cannot quantify the visibility of fingerprints; in fact, with equal contact angles, a fingerprint can be more or less visible depending on the optical properties of the surface. An easy-to-clean surface exhibits low wettability by the liquid, and therefore, the correspondent contact angle must be close to $90^{\circ}$ or higher. In this paper, a new approach is proposed for an objective classification method; a full characterization of various commercial surfaces for automotive interiors has been performed, and key properties are measured in correlation with the visibility of artificial fingerprints deposited with a standardized method.

\section{Material and Methods}

2.1. Materials. The 15 commercial surfaces for automotive interiors reported in Table 1(a) were tested. Different technologies of decoration were employed: 5 IMD, 5 IML, and 5 painted surfaces. Some of them were patterned (IMD1, IMD2, IMD3, and IML3), some transparent (IML2, IMD5), and some had a black finishing (IMD4, IML1, IML4, IML5, $\mathrm{V} 1, \mathrm{~V} 2, \mathrm{~V} 3, \mathrm{VN} 1$, and VN3).

Three of the painted surfaces were treated with a physical vapor deposition (PVD) coating layer to hopefully impart antifingerprint properties to the underlying surface (V1, $\mathrm{V} 2$, and V3).

The description of each surface with all relevant characteristics is shown in Table 1(a). The reagents for artificial fingerprints were all bought from Sigma-Aldrich and used without changes.

\subsection{Methods}

2.2.1. Reference Standard Fingerprint Preparation. A standard artificial fingerprint, representing an average human fingerprint, was developed to set up a surface classification based on the visibility of fingerprints in standard conditions.

To replicate the human fingerprint, both artificial sweat and sebum were required. The artificial sweat was prepared by dissolving the compounds listed in Table 2 (left column) in distilled water [9]. The weight $\%$ of the constituents listed in Table 2 compared to the complete formula in [9] is $\approx 80 \%$ $(\% w / w)$; this simplification is a good simulation of the real sweat with only 5 constituents easy to retrieve in the market. The complex mix of oils and fatty substances listed in Table 2 (right column) was blended to emulate the natural sebum; all are common oils or easy to recover constituents [10].

The mixture chosen to create the artificial fingerprints was composed of $95 \%$ sweat and 5\% sebum $(\% w / w)$. An aliquot of nonionic surfactant (Triton X-100) was required $(\approx 2 \mu \mathrm{l} \times$ grams of mixture $)$ to overcome the natural immiscibility between sweat and sebum.

2.2.2. Standard Fingerprint Deposition. The manual stamp for standard deposition was assembled by composing a stainless steel cylindrical support with a nut on the top (dimension: $M_{12}$ ) filled with cotton.

A layer of leather used in car interiors was pulled over to fix everything (Figure 1).

The pressure chosen to create the artificial fingerprint, in order to emulate a "soft touch" condition, was $\leq 3.5 \mathrm{~N} / \mathrm{cm}^{2}$. Since the assembled manual stamp had a contact area equal to $4 \mathrm{~cm}^{2}$, a $1 \mathrm{~kg}$ weight $(10 \mathrm{~N})$ was enough.

The deposition procedure was as follows:

(1) Heating the artificial secretion at $\approx 40^{\circ} \mathrm{C}$

(2) Withdrawing $1 \mu \mathrm{l}$ of heated artificial secretion with a micropipette $(20 \mu \mathrm{l})$

(3) Releasing the artificial secretion on the manual stamp and spreading it on the surface with a thin paint brush

(4) Positioning the manual stamp and the weight on the chosen surface

(5) Creating 3 consecutive artificial fingerprints spaced by $20 \mathrm{~mm}$ from each other

(6) Washing the manual stamp and the paint brush with distilled water, degreaser, and a cloth

2.2.3. Characterization Techniques. A Kruss DSA30S contact angle machine was used to measure the static apparent contact angle (CA) with distilled water (WCA) and diiodomethane $\left(\mathrm{CH}_{2} \mathrm{I}_{2} \mathrm{CA}\right)$. To prevent cross-contamination, a new syringe was used for each liquid. A drop of $0.5 \mu \mathrm{l}$ was used for the static contact angle; the image of the liquid drop 

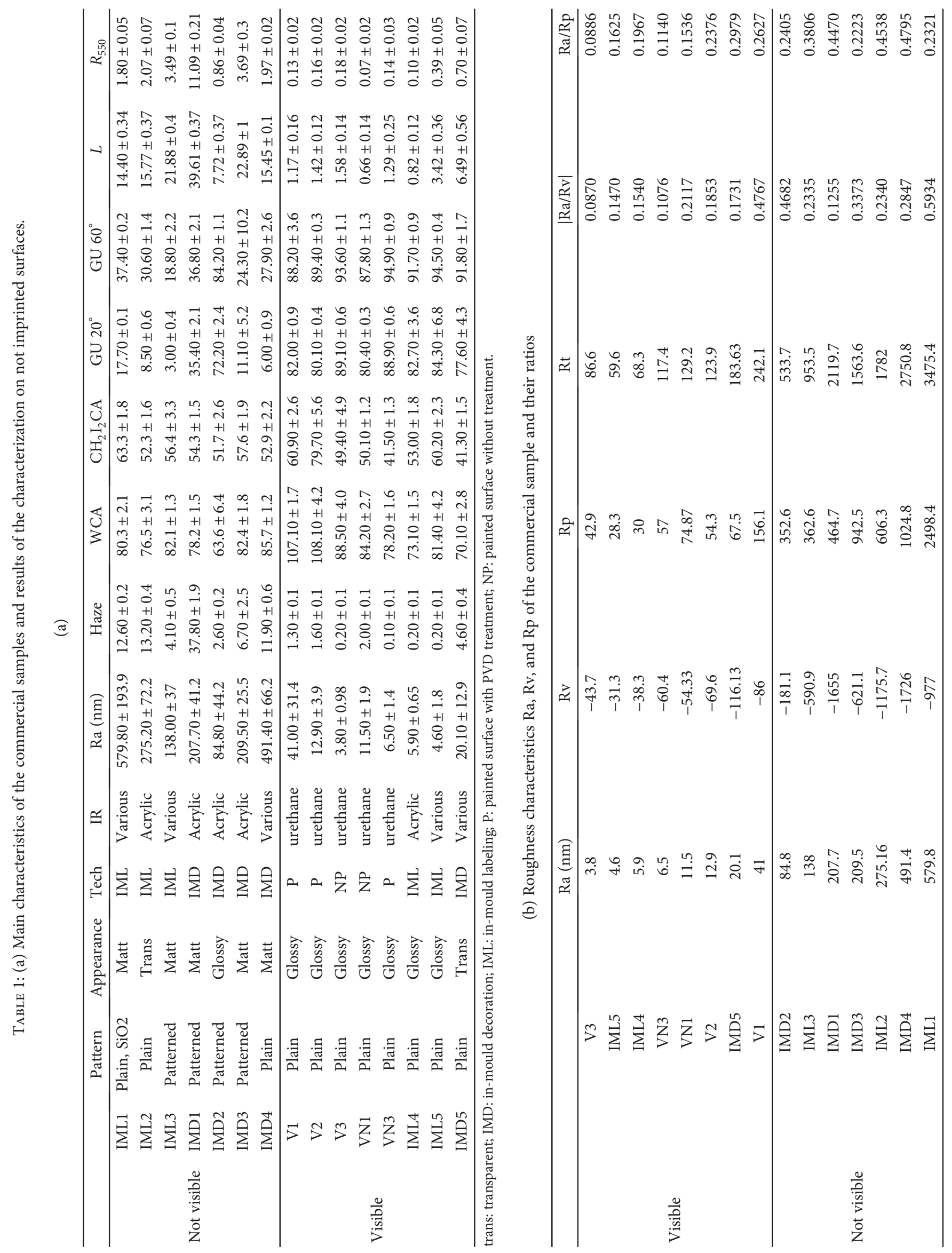
TABle 2: Components of the artificial sweat and their concentrations in $\mathrm{g} / \mathrm{l}$ and of artificial sebum and relative $\% \mathrm{w} / \mathrm{w}$.

\begin{tabular}{lccc}
\hline Artificial sweat & \multicolumn{2}{c}{ Artificial sebum } \\
\hline Reagent & $\mathrm{g} / \mathrm{l}$ & Reagents & $\%(w / w)$ \\
Lactic acid & 1.26 & Palmitic acid & 10 \\
$\mathrm{NaCl}$ & 1.53 & Stearic acid & 5 \\
$\mathrm{Urea}$ & 0.60 & Coconut oil & 15 \\
$\mathrm{KCl}$ & 0.46 & Paraffin wax & 10 \\
$\mathrm{NaHCO}_{3}$ & 0.25 & Jojoba oil & 15 \\
& & Olive oil & 20 \\
& & Squalene & 5 \\
& & Cholesterol & 5 \\
& & Oleic acid & 10 \\
\hline
\end{tabular}

was taken by a digital camera. Results are the average of 10 measures carried on each sample and for each liquid.

The surface roughness $(\mathrm{Ra})$ of all the samples was measured by a Bruker Dektak 150 stylus profilometer setting $2 \mathrm{mg}$ of weight, $400 \mu \mathrm{m}$ of scan length, $0.044 \mu \mathrm{m}$ of resolution, and $60 \mathrm{sec}$ of scan duration. $\mathrm{Rv}$ (the valley depth of the roughness profile below the mean line), $\mathrm{Rp}$ (the peak height over the mean line), and Rt (the distance between the highest peak and the deepest valley) were measured as well.

A Varian Cary 500 spectrophotometer was used for direct transmittance (T\%) measurements on glass, setting scans ranging from 300 to $800 \mathrm{~nm}$ and a scan rate of $600 \mathrm{~nm} / \mathrm{min}$.

IR spectra were acquired with a standard PerkinElmer Spectrum 100 (FT-IR) equipped with the ATR sampling system (PerkinElmer Universal ATR).

A Leica DCM8 confocal microscope was used to measure fingerprint thickness using a 20X magnification and an area of $1 \mathrm{~cm}^{2}$.

Optical characterization was performed with (a) Konica Minolta Rhopoint IQ Glossmeter for the GU and haze parameters, calibrated with the supplied standard, and (b) Konica Minolta 2600d portable spectrophotometer with an integrating sphere for reflectance $(R)$ and luminance $(L)$ parameters, using an $8 \mathrm{~mm}$ aperture and a scanning range from 360 to $740 \mathrm{~nm}$ in the SCE mode (specular component excluded). Reflection was measured at two reflection angles: $20^{\circ}$ (GU20) and $60^{\circ}$ (GU60). Two values were considered for $R$ : at $550 \mathrm{~nm}\left(R_{550}\right)$ and at the maximum difference along all the experimental range $\left(R_{\mathrm{MAX}}\right)$.

All parameters were measured before the deposition of fingerprints, so on cleaned surfaces and over the fingerprint mark after its deposition; in each case, at least 10 measures were collected (average values and errors are reported).

For any parameter $X(\mathrm{CA}, \mathrm{GU}$, haze, $R$, and $L)$, variation between the value on imprinted and on cleaned surfaces $(\Delta X \%)$ was calculated as follows:

$$
\Delta X \%=\left[\frac{\left(X_{1} \cdot 100\right)}{X_{0}}\right]-100 .
$$

According to the theory of error propagation, the new standard deviation $\sigma_{X}$ becomes

$$
\sigma_{X}= \pm\left[\frac{\sigma_{0}}{X_{0}}+\frac{\sigma_{1}}{X_{1}}\right] \cdot \frac{X_{1}}{X_{0}}
$$

where $\Delta X \%=\Delta \%$ is the variation of general parameter $X, X_{1}$ is the total average value on fingerprint spots, $X_{0}$ is the total average value on the cleaned surface, and $\sigma$ is the standard deviation, being $X$ a ratio between two different average values $X_{0} \pm \sigma 0$ and $X_{1} \pm \sigma 1$.

An optical microscope Nikon Eclipse LV100 was used to visually compare artificial and real fingerprints.

The principal component analysis (PCA) was carried out with XLSTAT 2015 software, autoscaling the variables according to the following formula:

$$
\left[\left(\frac{X 1-\mu 1}{\sigma_{1}}\right),\left(\frac{X 2-\mu 2}{\sigma 2}\right), \ldots,\left(\frac{X p-\mu p}{\sigma p}\right)\right],
$$

where $X 1$ is the value of parameter 1 for the single sample, $\mu 1$ is the average value for parameter 1 , and $\sigma$ is the standard deviation.

\section{Result and Discussion}

3.1. Comparison of Artificial and Human Fingerprints. Several measurements and comparisons were done on artificial and human fingerprints (deposited by 20 people: 10 men and 10 women) to verify if artificial fingerprints were a good approximation of the real ones.

An artificial fingerprint (the third one of the sequences deposited on the substrate) and a real fingerprint are compared in Figure 2; they look quite similar. The main difference is the pattern due to the leather used in one case and to the papillary crest in the other.

A further comparison was done by measuring transmittance $(T \%)$ on glass where a human fingerprint and three artificial fingerprints made with different secretion compositions $(95: 5,80: 20$, and $0: 100$, sweat/sebum $w \%)$ were deposited (Figure 3). The sebum decreases uniformly the transmittance in the range $400-800 \mathrm{~nm}$ : so, the higher is the amount of sebum, the lower is the $T \%$. This comparison supports the choice of the $95 \%(w / w)$ sweat and 5\% $(w / w)$ sebum mix as an optimal composition for the artificial fingerprint.

In addition, artificial and human fingerprints exhibit the same trend in modifying the wettability of a surface, as shown by contact angles measured with distilled water on a glass coated with Indio-Tin Oxide (ITO) (Figure 4).

Moreover, human and artificial fingerprints on glass behave similarly also with $R$ and haze parameters. Eventually, the thickness measured with a confocal microscope point outs, once more, to a close resemblance between artificial and real fingerprints (Table 3 ).

3.2. Visibility of the Standard Fingerprint on the Various Surfaces. After the deposition of fingerprints, all samples were observed by 5 people under artificial and natural light at different angles. In accord to their visual perception with 


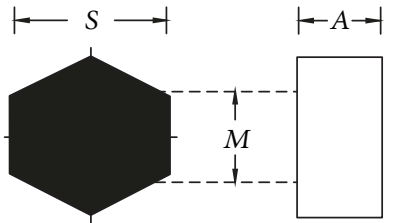

$M=12 \mathrm{~mm}$

$S=9 \mathrm{~mm}$

$A=10 \mathrm{~mm}$

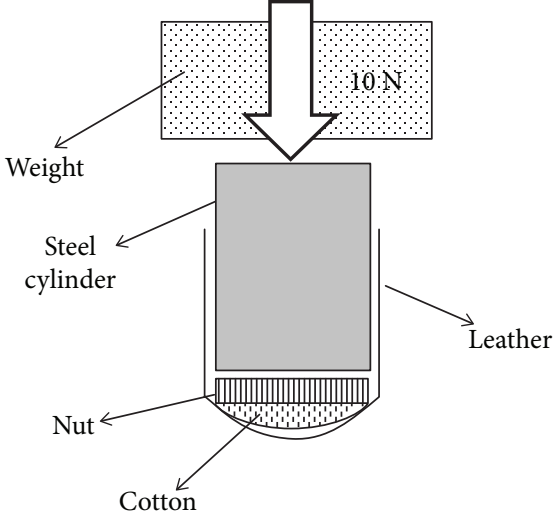

(b)

FIgURE 1: (a) View from the above and nut dimensions; (b) side view and stamp configuration.

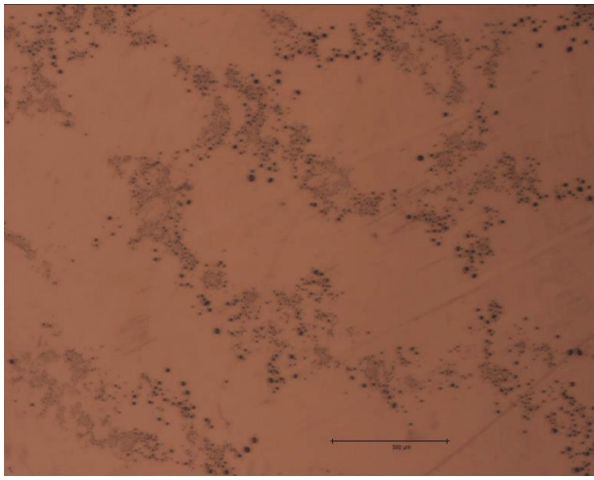

(a)

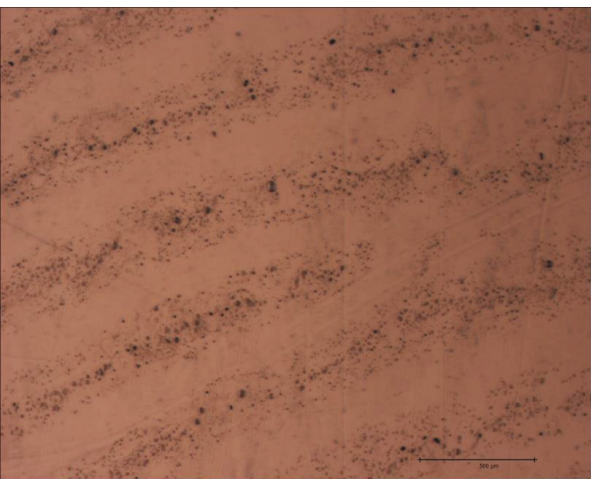

(b)

FIGURE 2: Images with optical microscope at 20X magnification of third artificial fingerprint (a) and a human fingerprint deposited by a man aging 25 years in low pressure condition (b).

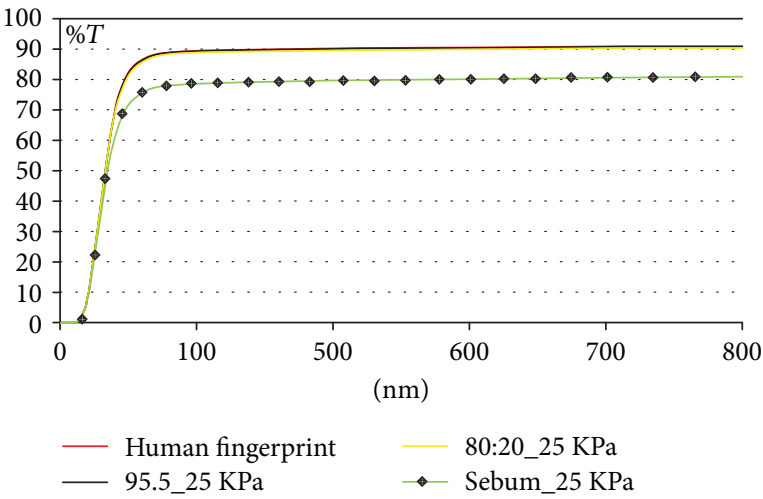

FIGURE 3: T\% spectra on glass with human and artificial fingerprints composed by different ratio \% sweat/sebum.

the naked eye, samples were divided into two categories: visible or nonvisible, as exemplified in Figure 5. This simple classification represents the customer perception.

There was not disagreement among the observers, and when a fingerprint was only partially visible, the sample was classified in the "visible" category.
The group of observers had no perception of fingerprints in the first seven samples of Table 1(a). The chemical structure of all surfaces was ascertained by ATR spectroscopy. Infrared spectra of the samples are reported in the supplementary materials in Figs S1, S3, and S3. Most of them can be grouped as acrylics or polyurethanes whilst some others pertain to a mixed group (Table 1(a)).

The results of the optical characterization, roughness, and measures of static contact angles on the cleaned surface for the whole set of samples are also reported in Tables 1(a) and 1(b).

The data of Table 1(a) evidence that in both categories, IMD and IML samples can be found; on the contrary, all painted surfaces examined were classified in the "visible" category, no matter if they received or not the antifingerprint treatment.

Apparently, there is no connection between an antifingerprint behavior and the technology of decoration or the polymer of which the surface is made.

On the contrary, glossiness, as measured by the reflection at $20^{\circ}$ or $60^{\circ}$, played a major role, since fingerprints were more visible on glossy surfaces than on matt ones. Moreover, the mark was more visible on smooth 


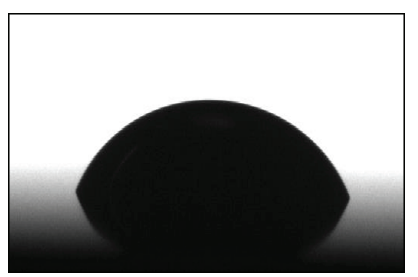

(a)

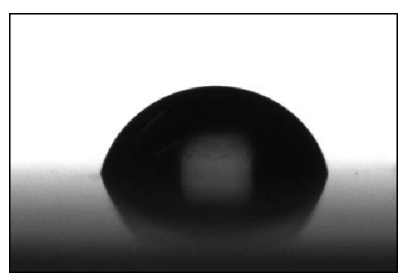

(b)

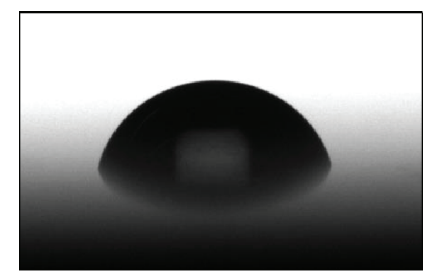

(c)

FIGURE 4: Images of water contact angles in different conditions: (a) glass/ITO $60.4 \pm 4.2$, (b) glass/ITO + real fingerprint $75.7 \pm 1.2$, (c) glass/ ITO + artificial fingerprint $75.6 \pm 3.3$.

TABle 3: Average thickness measurements with confocal microscope of artificial and human fingerprint.

\begin{tabular}{lc}
\hline Specimen & Average thickness $(\mu \mathrm{m})$ \\
\hline Human fingerprint & $0.25 \pm 0.02$ \\
Artificial fingerprint & $0.25 \pm 0.04$ \\
\hline
\end{tabular}

surfaces than on textured samples, no matter if they were glossy or matt.

In samples with high roughness $(\mathrm{Ra})$, haze, $L$, and $R_{550}$ and low GU, the fingerprint appearance tended to be optically masked; however, it was difficult to predict the limit over which this occurred and a specific cut-off value for each of these properties has to be found.

Apparent static contact angles in water (surface tension $0.073 \mathrm{Nm}^{-1}$ ) and in diiodomethane (surface tension $0.051 \mathrm{Nm}^{-1}$ ) did not correlate with the visibility of the standard fingerprint; for instance, the only two samples exhibiting low wettability (water contact angle $>90^{\circ}, \mathrm{V} 1$ and V2) appertained to the visible category. Absolute errors of contact angles measured on cleaned surfaces were relatively low (average values: water \pm 3 , diiodomethane \pm 2.4 corresponding to about 4\%). This suggests a sort of macroscopic homogeneity of the surfaces industrially prepared.

The apparent contact angle depends in a complex way on surface energies, roughness, and topography of the samples. Wu et al. [8] measured the contact angles on a series of chemically similar surfaces with different roughness $(\mathrm{Ra}<1 \mu)$ using an artificial liquid mimicking the biological one (surface tension $0.02-0.05 \mathrm{Nm}^{-1}$ ). By using the Cassie-Baxter approach [11], she found that surfaces with relatively deep valleys were able to trap air under the liquid droplet, improving the nonwetting behavior. Consequently, a new roughness factor for theoretical prediction of antifinger contact angles, $\mathrm{Ra} / \mathrm{Rv}<0.25$, was proposed, as a guideline for an easy-to-clean surface (contact angle $>90^{\circ}$ ). Smoother surfaces required even deeper valleys to impart antifingerprint property.

In Table 1(b), the full set of roughness parameters for the present commercial samples is reported $(\mathrm{Ra}<0.6 \mu)$. As a general trend, $\mathrm{Ra} / \mathrm{Rv}$ is larger for nonvisible samples (up to 0.59 ) than for visible ones (up to 0.21). Thus, fingerprints do appear on surfaces with very low $\mathrm{Ra} / \mathrm{Rv}$, which could be considered "easy to clean" according to Wu's findings, and fingerprint marks do not appear on surfaces with relatively shallow valleys. However, Wu measures were carried out on a series of homogenous laboratory-prepared surfaces whilst in the present case, surfaces prepared with a number of different industrial techniques and with a large variety of materials (labels, paints, etc.) were investigated, on which other factors play a significant role in the resulting apparent contact angle. In addition, a relatively good negative correlation between $\mathrm{Rp}$ (peak value) and $\operatorname{cosCH}_{2} \mathrm{I}_{2} \mathrm{CA}$ was found $\left(R^{2} 0.7019\right)$ for samples of the nonvisible category, suggesting a positive effect of a bumpy structure in finger-free surfaces. Further investigations were carried out on imprinted surfaces to better clarify the reason for appearance of fingerprint marks.

3.3. Imprinted Surface Characterization. In order to correlate the visibility of the fingerprint with the optical properties of the chosen surfaces, the same measurements were performed directly on the artificial fingerprint deposited on the samples. The \% differences found in the two sets of measurements (imprinted and not imprinted surfaces) were calculated and reported in Table 4, together with the original value of roughness Ra. Absolute error of contact angles was larger when measured on imprinted surfaces than on cleaned surfaces (average values on cleaned surfaces: water \pm 3 , diiodomethane \pm 2.4 ; on imprinted surfaces: water \pm 4.7 , diiodomethane \pm 3.1 ). This suggests that measurements on fingerprint marks were affected by a larger experimental error those than on cleaned surfaces, even if standard deposition of the liquid was performed. 


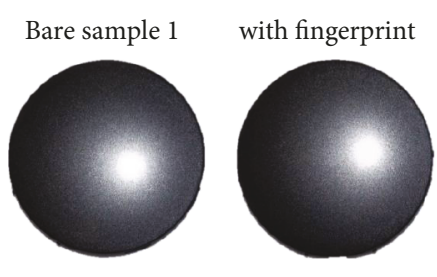

Nonvisible

(a)

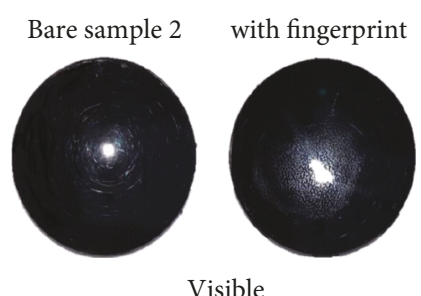

(b)

FIgURE 5: IML1 (a) and IML5 (b) views before and after the deposition of the artificial fingerprint.

When fingerprint was visible with the naked eyes, $\Delta$ haze, $\Delta L, \Delta R_{550}$, and $\Delta R_{\mathrm{MAX}}$ dramatically increased; on the contrary, $\Delta \mathrm{GU}$ (both $20^{\circ}$ and $60^{\circ}$ ) and $\Delta \mathrm{CA}$ tended to decrease. This occurred in all "visible" samples apart from transparent IMD5 where $\Delta \mathrm{GU}$ slightly increases. All these results demonstrate that fingerprint application makes a surface less brilliant.

"Nonvisible" samples performed somewhat differently: $\triangle \mathrm{GU}$ and $\triangle \mathrm{WCA}$ had a contrasted behavior, and $\Delta$ haze, $\Delta L, \Delta R$, and $\Delta R_{\mathrm{MAX}}$ were hardly affected; however, $\Delta \mathrm{CH}_{2}$ $\mathrm{I}_{2} \mathrm{CA}$ decreased suggesting that fingerprint deposition on the substrate occurred in nonvisible cases also but that their appearance was masked by optical diffusion. Haze highly increased only in the patterned glossy IMD2 sample.

Thus, the appearance/nonappearance of fingerprint marks seems to depend on several properties. A comprehensive approach was performed by making a PCA on the data of Table 4 after they were autoscaled (to make measures of different units and range comparable each other).

A relatively good separation between the two classes appears in the score plot (Figure 6(a)); however, the corresponding loading plot (Figure 6(b)) puts in evidence the overlapping contribution of some variables, which therefore gave the same information ( $\triangle \mathrm{GU} 20^{\circ}$ and $\Delta \mathrm{GU} 60^{\circ}$, $\Delta L \%$ and $\Delta R \%)$. At the same time, other variables notably $\Delta \mathrm{WCA}$ and $\Delta \mathrm{CH}_{2} \mathrm{I}_{2} \mathrm{CA}$ seem to be less significant despite that contact angles are reported to be crucial to evaluate antifingerprints properties [8, 11]. An explanation could be, as reported in literature [12], that the apparent static contact angles that we measured are less correlated to the quality of the surfaces than the advancing and receding dynamic contact angles.

However, the variation of the static contact angles measured on the fingerprint mark and on the clean surface was under consideration here $\left(\triangle \mathrm{WCA}\right.$ and $\left.\Delta \mathrm{CH}_{2} \mathrm{I}_{2} \mathrm{CA}\right)$ and not the contact angles themselves. From Table 4 , it can be recognized that there is a general decreasing of $\Delta \mathrm{CA}$ (both in water and in diiodomethane) when measured on the fingerprint mark (visible or not visible) instead of on the cleaned surface. So, even in the case of not visible fingerprint surfaces, some residual material from the finger liquid sticks on the surface making it apparently less hydrophilic. A better knowledge on the oleophobic properties of the investigated surface can be gained by measuring the contact angles by using a probing liquid with a lower surface tension, such as oleic acid (which is one of the main components of the artificial sebum).
All these considerations can explain why the measured static contact angles do not give a significant information in the PCA treatment.

Consequently, a PCA was performed on a reduced number of variables. The results (Figure 7) indicated that the reduction of the variable number did not impair the separation between visible and nonvisible samples.

All "nonvisible" samples were confined in a narrow area of the negative quadrant of the plot where $\mathrm{Ra}$ and $\Delta \mathrm{GU}$ at $20^{\circ}$ were dominant, except the IMD2 sample (patterned, glossy). Visible samples were spread over the other quadrants where $\Delta R_{\mathrm{MAX}}, \Delta L$, and $\Delta$ haze were dominant apart for the IMD5 sample (transparent, glossy) which fell close to the zone of "nonvisible" samples.

Such classification is good, but more attention is required for transparent and patterned samples.

3.4. Analytical Method for Antifingerprint Properties. The separation of the two categories of samples (fingerprint visible-nonvisible) was good enough even reducing the number of variables to five (and consequently the number of measures, specifically $\mathrm{Ra}, \Delta \% \mathrm{GU} 20^{\circ}, \Delta \%$ haze, $\Delta \%$ $\left.R_{\mathrm{MAX}}, \Delta \% L\right)$.

A classification method to evaluate and quantify the ability of optically masking fingerprints on chosen surfaces is proposed, based on cut-off values; for most significant parameters, an average value between the limit of each series (visible and not visible) was estimated from the data of Table 4 and taken as cut-off value. The anomalous value for $\Delta$ haze $\%$ for IMD2 was disregarded in this calculation. The selected cut-off values are reported in Table 5, first row.

Further, the ability to emulate human fingerprints was checked by repeating the measurements of the four parameters ( $\mathrm{Ra}$ excluded) on four samples using the real human fingerprints of 20 people (10 men and 10 women) in "soft-touch" condition. At least one surface for each technology and two surfaces for each category were selected for this purpose. The proposed cut-off values provided the right classification of the surfaces also when imprinted with human fingerprints (Table 5, right). However, the parameter variation was generally larger with human than with artificial fingerprints, possibly owing to the difficulty to replicate the standard deposition conditions manually (applied pressure) and the possible presence of contaminants.

Eventually, measurements of the five significant parameters were repeated on two samples not previously used in 


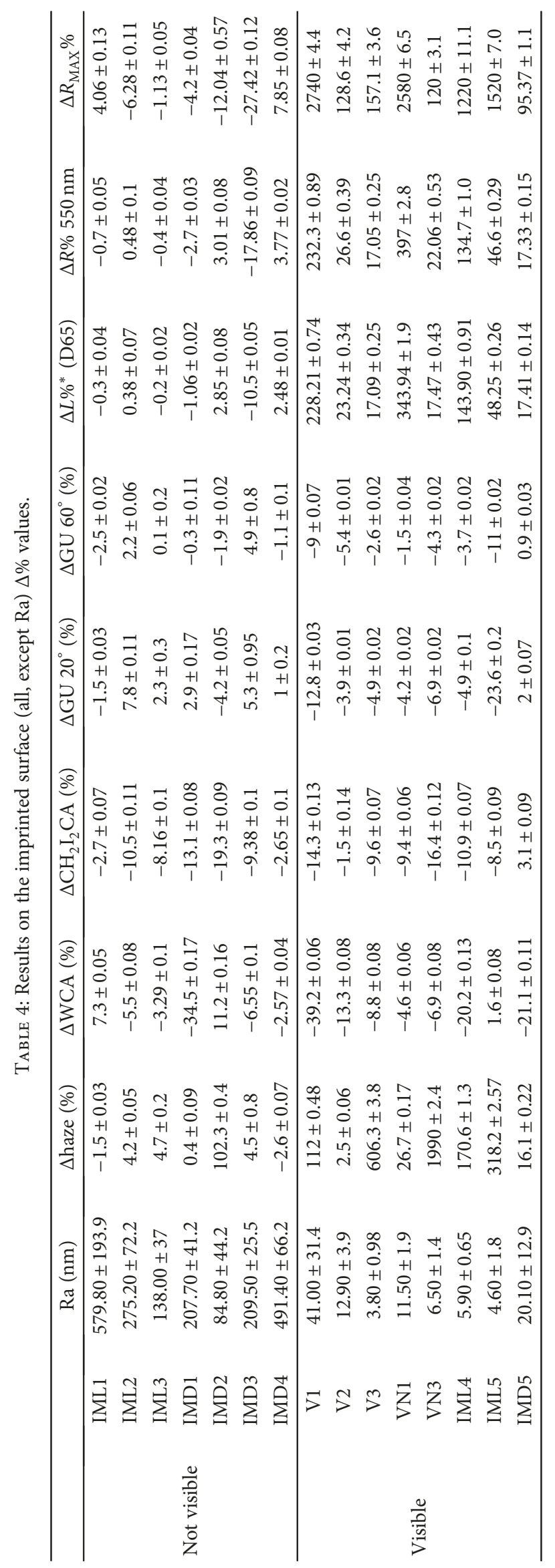




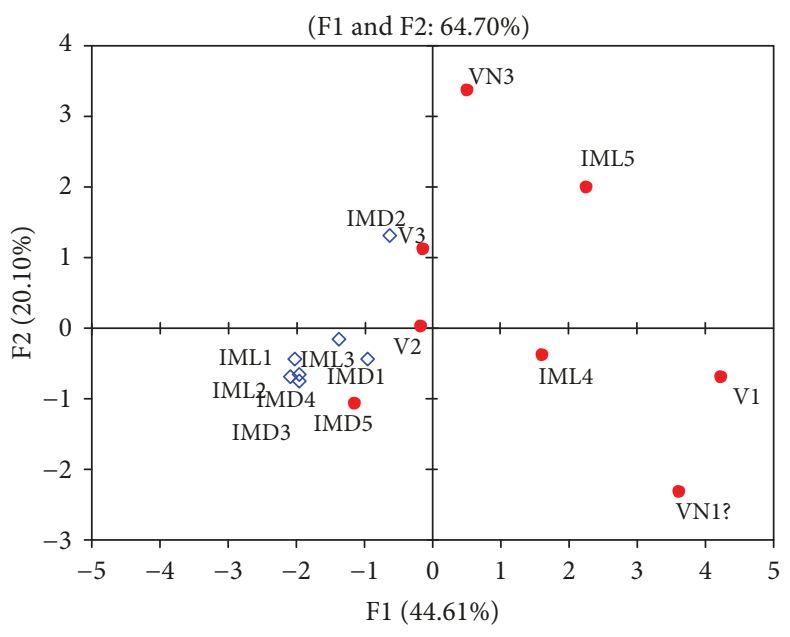

(a)

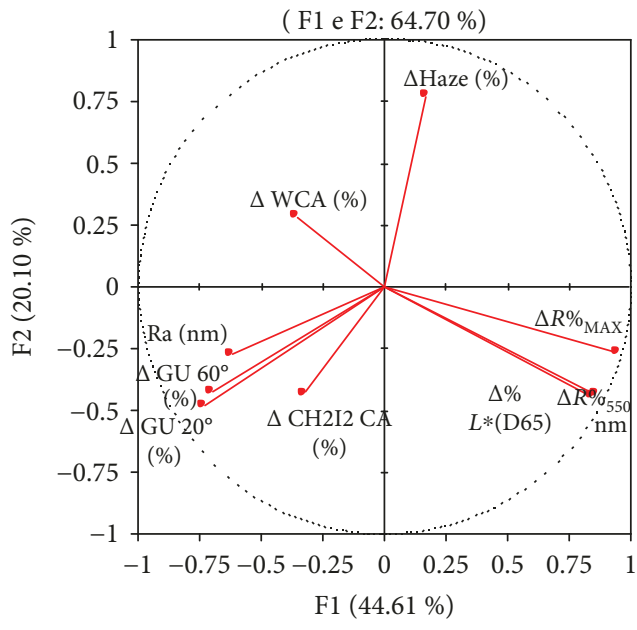

(b)

FIGURE 6: Score and loading plots from PCA performed with all variables. Score plot: (a) closed red marks represent "visible" and empty marks represent "nonvisible." Loading plot (b).

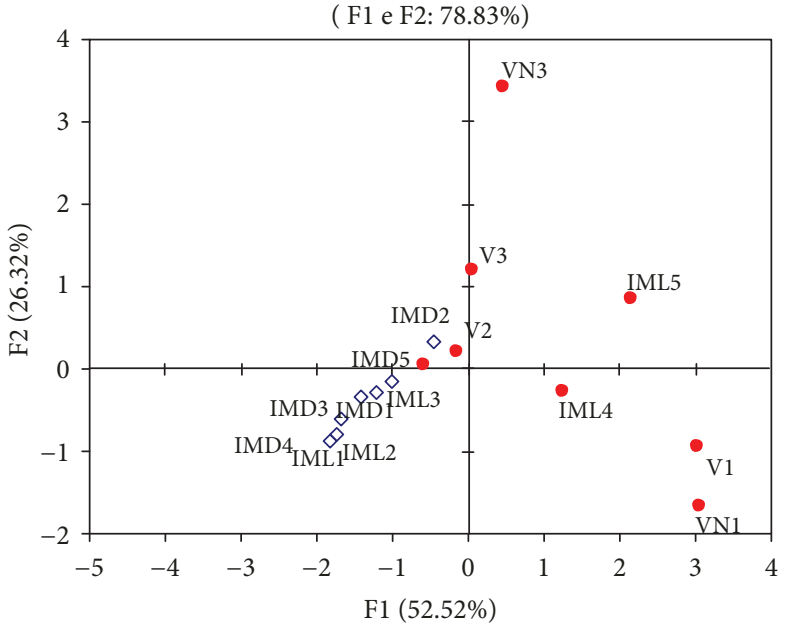

(a)

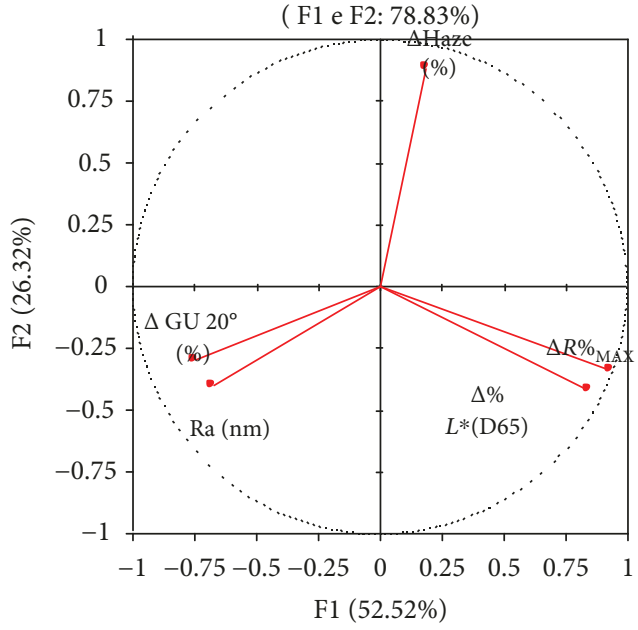

(b)

FIGURE 7: Score and loading plots from PCA performed with a reduced number of variables. Score plot: (a) closed red marks represent "visible" and empty marks represent "not visible." Loading plot (b).

TABLE 5: Cut-off values and average results of the four significant parameters on selected surfaces using artificial and human fingerprint.

\begin{tabular}{|c|c|c|c|c|c|c|c|c|}
\hline & \multicolumn{4}{|c|}{ Artificial fingerprint } & \multicolumn{4}{|c|}{ Human fingerprint } \\
\hline & $\Delta \%$ haze & $\Delta \% \mathrm{GU} 20^{\circ}$ & $\Delta \% L$ & $\Delta \% R_{\mathrm{MAX}}$ & $\Delta \%$ haze & $\Delta \% \mathrm{GU} 20^{\circ}$ & $\Delta \% L$ & $\Delta \% R_{\mathrm{MAX}}$ \\
\hline Cut-off & 10 & -2 & 10 & 50 & 10 & -2 & 10 & 50 \\
\hline IML2 & $4.2 \pm 0.05$ & $7.8 \pm 0.11$ & $0.38 \pm 0.07$ & $-6.28 \pm 0.11$ & $5.18 \pm 0.1$ & $2.37 \pm 0.1$ & $-0.08 \pm 0.03$ & $7.88 \pm 0.14$ \\
\hline IMD1 & $0.4 \pm 0.09$ & $2.9 \pm 0.17$ & $-1.06 \pm 0.01$ & $-4.20 \pm 0.04$ & $6.24 \pm 0.1$ & $14.69 \pm 0.1$ & $-2.87 \pm 1$ & $-5.68 \pm 1$ \\
\hline VN3 & $1990 \pm 2.4$ & $-6.9 \pm 0.2$ & $17.47 \pm 0.43$ & $120 \pm 3.1$ & $2075 \pm 64.8$ & $-15.59 \pm 0.2$ & $94.06 \pm 0.7$ & $616 \pm 10.7$ \\
\hline IML4 & $170.6 \pm 1.3$ & $-4.9 \pm 0.1$ & $143.90 \pm 0.9$ & $1220 \pm 11.1$ & $540 \pm 5.8$ & $-12.66 \pm 0.1$ & $64.75 \pm 0.4$ & $1236 \pm 11.8$ \\
\hline
\end{tabular}

order to evaluate the prediction ability of the method. The classification was done by using the proposed cut-off values and then confirmed with naked eye observation (Table 6).

The samples were classified correctly in their respective categories, although some parameters (highlighted in bold) have a dubious value.

\section{Conclusions}

This work was aimed at elaborating a classification method to objectify the visibility of fingerprints on surfaces, instead of the evaluation of the "easy-to-clean" properties already used. 
TABLE 6: Average results of significant parameters for the selected surfaces and relative classification.

\begin{tabular}{|c|c|c|c|c|c|c|c|c|c|}
\hline & $\mathrm{Ra}(\mathrm{nm})$ & $\Delta$ haze $(\%)$ & $\Delta \mathrm{GU} 20^{\circ}(\%)$ & $\Delta \% L$ & $\Delta \% R_{\mathrm{MAX}}$ & Visibility & Pattern & Appearance & Tech \\
\hline Cut-off & 50 & 10 & -2 & 10 & 50 & & & & \\
\hline VN2 & $613.07 \pm 39.5$ & $33.33 \pm 1.1$ & $15.15 \pm 0.47$ & $-0.87 \pm 0.01$ & $-2.42 \pm 0.03$ & Not visible & Plain & Matt & $\mathrm{NV}$ \\
\hline IMD6 & $47.14 \pm 9.9$ & $33.71 \pm 0.16$ & $-5.90 \pm 0.03$ & $58.27 \pm 0.5$ & $716.00 \pm 8.14$ & Visible & Plain & Glossy & IMD \\
\hline
\end{tabular}

An artificial fingerprint liquid consisting in artificial sebum and artificial sweat $(5: 95 \% w / w)$ was prepared and deposited on the surfaces in selected standard conditions: both liquid and standard conditions were easily replicable. The similarity of artificial and real human fingerprints was confirmed, and the standard deposition method was shown to eliminate the substantial variability of human fingerprints, offering high reproducibility. The visibility of fingerprints seems to depend on several properties; however, a comprehensive chemometric approach allowed to reduce the significant measurements to 5 properties: the roughness profile $(\mathrm{Ra})$, the \% variation of gloss $(\mathrm{GU})$, haze, luminance $(L)$, and diffuse reflectance $(R)$.

This restricted number of variables allowed to classify a surface correctly by using a method based on cut-off values, and the results of the measurements permitted to quantify the antifingerprint optical properties of the surface. The value-added feature of this work is of course the objectification of the visibility of fingerprints on chosen surfaces, instead of the common naked-eye evaluation.

From data acquired, it is evident that rough and matt surfaces promote the optical masking of fingerprints, whilst chemical treatment intended to decrease wettability and reduce adhesion of the fingerprint on the surface was not always successful, confirming that most of commercially treatments available are focused on improving the "easyto-clean" properties. Glossy surfaces are critical for the point of view of antifingerprint properties; however, an appropriate pattern could be a useful trick in order to hide fingerprints or reduce their visibility. Care should be adopted with transparent surfaces, where roughness and reflectance seem to govern the antifingerprint properties. With future efforts in increasing the number of samples studied, the cut-off values will be more reliable. Moreover, measuring the dynamic contact angles instead of static ones and using a probing liquid with surface tension approaching that of artificial fingerprint liquid will further improve the analysis results, providing a more detailed design guideline for producing visually fingerprint-free surfaces.

\section{Data Availability}

The data on contact angle, surface roughness, transmittance, gloss, haze, reflectance, and luminance parameters, used to support the findings of this study, are included within the article. The FTIR spectra data used to support the findings of this study are included within the supplementary information file; original files are available from the corresponding author upon request.

\section{Conflicts of Interest}

The authors declare that they have no conflicts of interest.

\section{Acknowledgments}

The authors wish to thank Dr. M.M. Dugand for the most helpful discussions and for performing confocal analysis. FCA sponsors this work by carrying out optical characterizations and supporting V.P. fellowship.

\section{Supplementary Materials}

Figure S1: IR spectra of samples IML2, IML3, IMD1, IMD2, IMD3, and acrylic polymers. Figure S2: IR spectra of V3, VN3, V2, V1, VN1, and polyurethane polymers. Figure S3: IR spectra of IML1, IML4, IML5, IMD5, and IMD4 various polymers. (Supplementary Materials)

\section{References}

[1] P. Uhlmann, R. Frenzel, B. Voit et al., "Research agenda surface technology: future demands for research in the field of coatings materials," Progress in Organic Coatings, vol. 58, no. 2-3, pp. 122-126, 2007.

[2] B. Stoehr, S. McClure, A. Höflich et al., "Unusual nature of fingerprints and the implications for easy-to-clean coatings," Langmuir, vol. 32, no. 2, pp. 619-625, 2016.

[3] A. Girod, R. Ramotowski, and C. Weyermann, "Composition of fingermark residue: a qualitative and quantitative review," Forensic Science International, vol. 223, no. 1-3, pp. 10-24, 2012.

[4] S. Cadd, M. Islam, P. Manson, and S. Bleay, "Fingerprint composition and aging: a literature review," Science and Justice, vol. 55, no. 4, pp. 219-238, 2015.

[5] T. Jiang, Z. Guo, and W. Liu, "Biomimetic superoleophobic surfaces: focusing on their fabrication and applications," Journal of Materials Chemistry A, vol. 3, no. 5, pp. 1811$1827,2015$.

[6] B. C. Wilson and D. J. Fiore, "Anti-fingerprint coating applications for automotive touchscreen displays," in SID Symposium Vehicle Displays \& Interfaces, p. 204, Dearborn, MI, USA, October 2011.

[7] A. Siriviriyanun and T. Imae, "Anti-fingerprint properties of non-fluorinated organosiloxane self-assembled monolayercoated glass surfaces," Chemical Engineering Journal, vol. 246, pp. 254-259, 2014.

[8] L. Y. L. Wu, S. K. Ngian, Z. Chen, and D. T. T. Xuan, "Quantitative test method for evaluation of anti-fingerprint property of coated surfaces," Applied Surface Science, vol. 257, no. 7, pp. 2965-2969, 2011. 
[9] C. J. Harvey, R. F. LeBouf, and A. B. Stefaniak, "Formulation and stability of a novel artificial human sweat under conditions of storage and use," Toxicology In Vitro, vol. 24, no. 6, pp. 1790-1796, 2010.

[10] J. L. Staymates, M. E. Staymates, and G. Gillen, "Evaluation of a drop-on-demand micro-dispensing system for development of artificial fingerprints," Analytical Methods, vol. 5, no. 1, pp. 180-186, 2013.

[11] M. Belhadjamor, M. el Mansori, S. Belghith, and S. Mezlini, "Antifingerprint properties of engineering surfaces: a review," Surface Engineering, vol. 34, no. 2, pp. 85-120, 2018.

[12] H. B. Eral, D. J. C. M. 't Mannetje, and J. M. Oh, "Contact angle hysteresis: a review of fundamentals and applications," Colloid and Polymer Science, vol. 291, no. 2, pp. 247-260, 2013. 


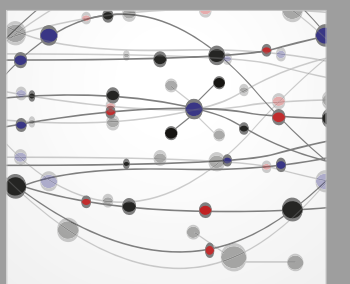

The Scientific World Journal
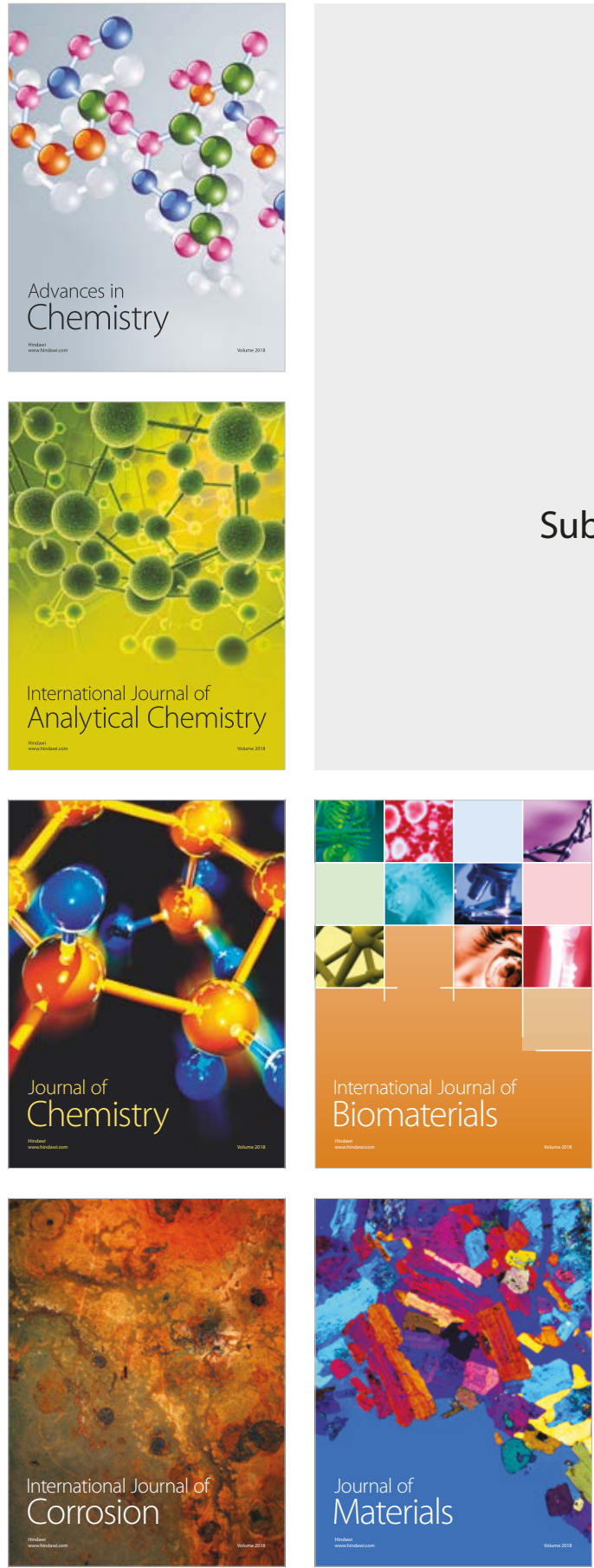

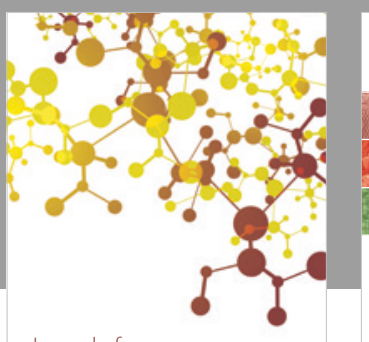

Journal of

Applied Chemistry
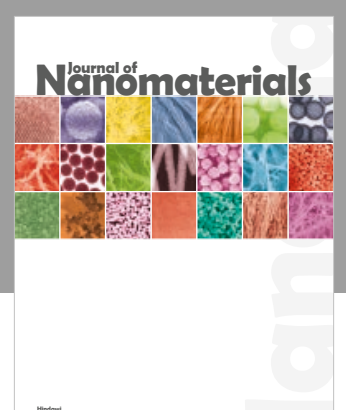

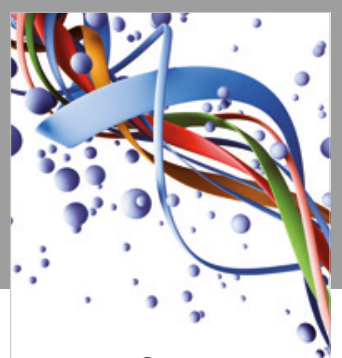

Scientifica

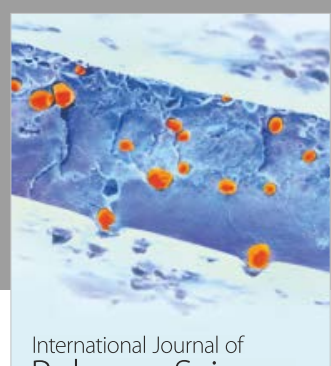

Polymer Science

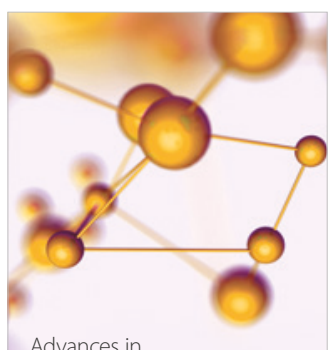

Physical Chemistry
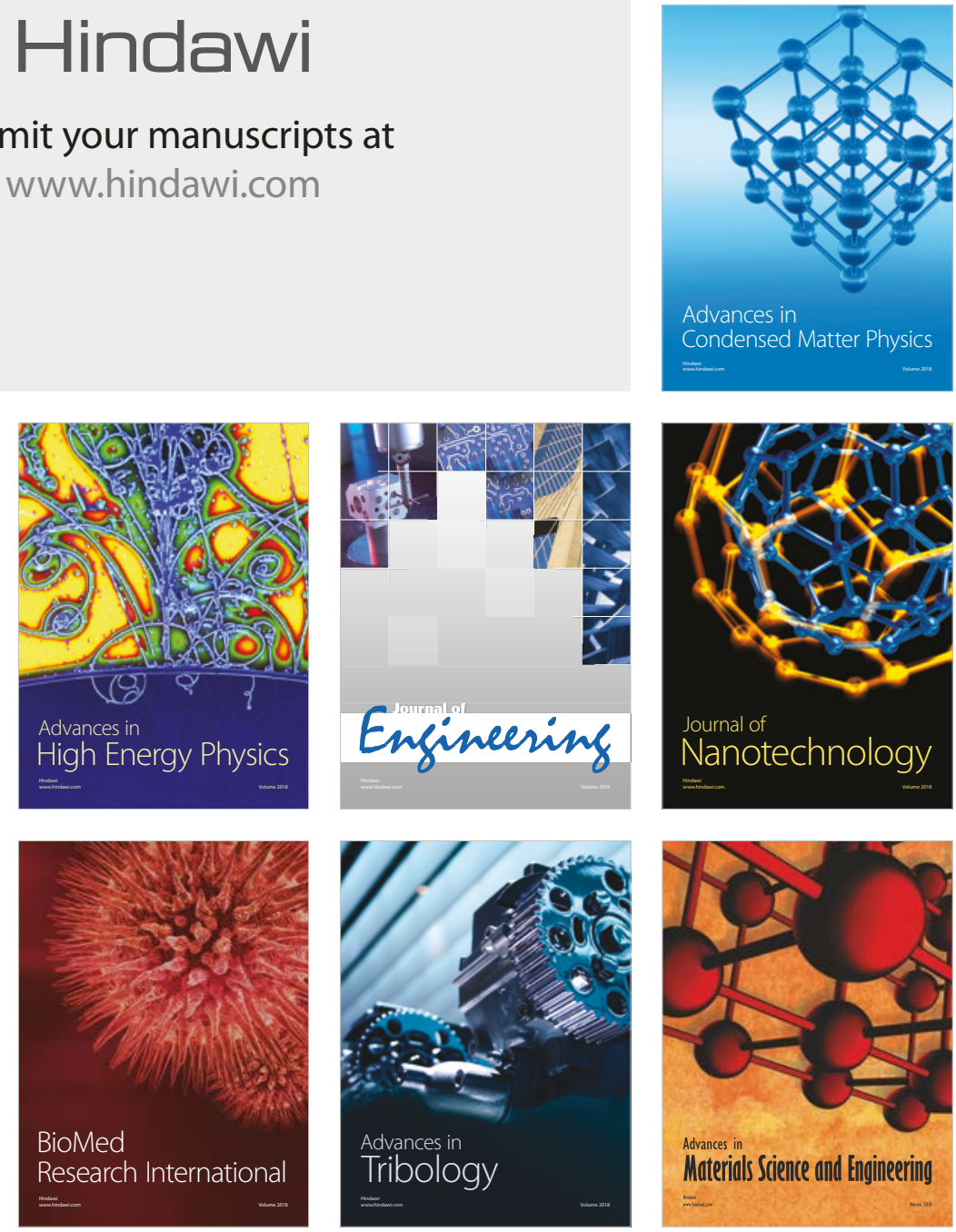